Meta

Journal des traducteurs

Translators' Journal

\title{
La Loi 22 et la traduction
}

\section{Michel Guillotte}

Volume 21, numéro 1, mars 1976

La traduction et l'entreprise

URI : https://id.erudit.org/iderudit/002990ar

DOI : https://doi.org/10.7202/002990ar

Aller au sommaire du numéro

Éditeur(s)

Les Presses de l'Université de Montréal

ISSN

0026-0452 (imprimé)

1492-1421 (numérique)

Découvrir la revue

Citer cet article

Guillotte, M. (1976). La Loi 22 et la traduction. Meta, 21(1), 95-99.

https://doi.org/10.7202/002990ar

Ce document est protégé par la loi sur le droit d'auteur. L'utilisation des services d'Érudit (y compris la reproduction) est assujettie à sa politique d'utilisation que vous pouvez consulter en ligne.

https://apropos.erudit.org/fr/usagers/politique-dutilisation/
Cet article est diffusé et préservé par Érudit.

Érudit est un consortium interuniversitaire sans but lucratif composé de l’Université de Montréal, l'Université Laval et l'Université du Québec à Montréal. Il a pour mission la promotion et la valorisation de la recherche. https://www.erudit.org/fr/ 


\section{La Loi 22 et la traduction}

Passé l'effet de surprise, de nouveauté et de publicité outrancière, la Loi 22 se trouve remise à sa juste place dans un ensemble d'événements non concertés. Pour l'entreprise, la réaction qui se déclenche ramène l'événement législatif à sa juste proportion. Si l'heure des bilans n'est pas encore sonnée, les manifestations les plus tapageuses de l'actualité laissent place à l'interrogation. Dans la balance prennent place en ce moment de multiples contrepoids qui distinguent la traduction comme l'un des avatars de l'éternelle poursuite de la francisation du Québec, des politiques régionales et des politiques continentales.

S'il n'y avait pas péril en la demeure, il faudrait laisser jeter sa gourme à la nouvelle génération, poussée pendant la réforme scolaire, grandie dans le culte d'un Québec aux Québécois que les aînés eussent aimé pratiquer plus pacifiquement. Et même, certains de ceux que l'on affiche d'ordinaire pour libéraux au sens large du terme se gardent bien de donner dans le panneau au-delà d'un affichage qui n'est pas toujours de bon aloi! Bref, le label linguistique, qui n'a jamais spécialement séduit les hommes d'affaires, s'éloigne en vérité comme en valeur. Les événements des dix ou quinze dernières années permettent d'en juger.

Toute réflexion sur l'évolution de la traduction dans l'entreprise depuis les années soixante part en fait de la loi fédérale sur les langues officielles. Pendant longtemps, il a été admis comme un dogme que le traducteur était un homme qui, en mettant le doigt au dictionnaire, appréhendait des mots qu'il n'avait plus qu'à transcrire et, lorsqu'il était doublé d'un terminologue, à classer. Or, quelle est la relation entre la loi fédérale et le traducteur? On peut la comparer au point en géométrie. De même que le point ne possède aucune valeur en soi, qu'il n'est que le résultat d'une opération intellectuelle par laquelle des lignes imaginaires se coupent, qu'il ne possède de signification qu'en fonction des lignes dont il définit l'intersection, la loi sur les langues officielles n'est que la conséquence d'une convergence de courants socio-politiques.

La politique fédérale était l'expression d'un cas de conscience, car comment enseigner l'harmonie nationale sans se demander ce que cela signifiait pour les Canadiens français, obligés de vivre tous les jours par procuration. L'histoire a donc rendu la justice à sa manière : elle a doté le gouvernement fédéral d'abord, les grandes entreprises traitant au Québec ensuite, de l'arme de la traduction. 
Déjà done, avant 1970 , les traducteurs se formaient en cohortes au sein des grandes entreprises et, s'y promenant comme des chiffonniers, trouvaient à chaque pas quelque chose à mettre dans leur hotte, suivant le principe qui veut que lorsqu'on ne sait pas ce qu'on cherche, on ne sait pas ce qu'on trouve.

La grande affaire du chef de service, à cette époque héroïque comme d'ailleurs trop souvent maintenant encore, était de distinguer les besoins réels et de s'en tenir à ceux-ci. Il lui fallait contenir les assauts de ceux qui voulaient tout traduire à tout prix. La découverte était belle : il fallait rattraper en quelques mois deux siècles d'obscurantisme, sans égard à la qualité.

Pour traduire correctement tous ces mots, il aurait fallu, quand on les rencontrait pour la première fois, faire attention à leur graphie au lieu de les survoler en pensant à ce parfum d'à quoi bon que dégagent trop de Canadiens français lorsqu'il s'agit de l'usage de leur langue. Nous vivons à une époque caractérisée par l'éparpillement et le goût du moindre effort, et nous parlons une langue qui figurait il y a peu comme le huitième péché capital ; comment s'étonner dès lors qu'en élevant soudain cette langue jusqu'aux nues, il se trouve encore des gens pour juger le sujet trop abstrait, trop immatériel, trop ambitieux. Au travail, où il s'efforçait traditionnellement d'acquérir le vocabulaire puis la mentalité de son collègue anglais, l'employé francophone aurait cru gaspiller ses facultés intellectuelles et prostituer son intelligence en apprenant en quoi chariot diffère de charrette, attraper de trappe et bonhomie de bonhomme. Trop souvent, une vigilance armée opposait le Canadien français de l'entreprise à ses compatriotes du service de traduction, et sa fierté d'être francophone se bornait à s'enquérir avec aménité de l'identité linguistique du contrôleur de chemin de fer ou de l'hôtesse de ligne aérienne.

\section{LA LOI 22 OU L'INCONFORT INTELLECTUEL}

Elle vint quelques années plus tard, la Loi 22, comme l'intendance suit les armées. En fait, elle suivait la voie toute tracée de la sensibilisation des entreprises à l'existence du marché français au Canada. Pour certains, elle représente le sommet de l'acquisition civilisatrice, le difficile aboutissement de l'aventure française en Amérique, le symbole de la souveraineté culturelle en face des servitudes de l'histoire. Pour d'autres, elle représente les délires et les modes d'une caste enfermée dans ses intérêts. Connaissant déjà le réservoir de bonne volonté des entreprises à l'endroit du fait français, j'aurais aimé que l'un des soucis du législateur, hormis la promotion des cadres francophones, fût d'apprendre aux Québécois eux-mêmes à aimer leur langue et, pour ce faire, de provoquer le rapprochement entre ceux qui ont pour métier de la bien connaître et ceux qui ont pour destin de la parler. Le traducteur est cet homme singulier qui, depuis des années, n'a cessé d'irriter tour à tour les apôtres du laisser braire, les administrateurs illettrés, les techniciens paresseux et les écrivains joualisants et auquel pourtant nous devrions accorder une fonction purificatrice, si l'on en juge par la place qu'il tient dans la production de textes français dans l'entreprise au Québec. 
Ce qui frappe d'abord en cette loi, c'est son côté navigateur. Impression première qui doit peut-être quelque chose au fait qu'elle a trouvé habitat à l'extrême pointe des préoccupations socio-économiques, là où quatre rives n'en font plus que deux et où le regard ne remonte pas le courant mais file avec lui vers quelque imprécise et permanente structure. Impression de malaise, aussi, qui vient de ce que les règles du jeu demeurent inconnues, mal bien pire pour l'entreprise et pour l'individu qu'une ligne de conduite arrêtée et conforme à un dessein connu et compris de tous.

\section{LES RÉPERCUSSIONS DE LA LOI 22 POUR L'ENTREPRISE ET LA TRADUCTION}

À ce stade-ci de l'évolution de la traduction dans l'entreprise au Québec, une sensibilité nouvelle affronte une structure vieillie. Entre les traducteurs désireux de trouver une dimension nouvelle à leur métier et les entreprises figées, malgré elles, dans l'attente des règles du jeu, le débat se réduit très vite à l'appel à la corvée des premiers, aux frais des secondes. Or, dans une perspective globale, c'est l'utilisation originale du français qui est au cour des discussions sur le sens de la traduction. La nécessité qu'il y a de franciser les entreprises au Québec fait d'une activité longtemps considérée comme imitative et parasite l'instrument privilégié de la conception en français. Non plus seulement traduire, mais aider à rédiger directement en français, non plus traduire à grands frais pour l'entreprise, mais participer à la formation d'employés qui, demain, n'auront plus à faire le chemin de Canossa du service linguistique pour mettre au point la moindre note de service.

Depuis longtemps, on reconnaît volontiers aux traducteurs deux fonctions principales dans l'entreprise. D'abord, une fonction d'information : le traducteur fournit les mots qui éclairent le travail de ses collègues des autres services. Cette activité, liée au développement de la complexité technologique et à la mode des communications, répond à l'appétit de curiosité immédiate des autres employés. Elle joue également le rôle d'un tampon, protégeant l'employé contre le choc d'une rencontre trop brutale avec le terme exact. Vient ensuite une fonction de transposition : le traducteur met fidèlement en français un texte dans sa forme, son contenu et son environnement culturel d'origine. Cette pratique permet d'obtenir la preuve apaisante que la création d'un texte procède toujours d'une intelligence extérieure, à laquelle le français n'a plus qu'à s'adapter fidèlement. Ainsi, en fonction de ce double rôle, le traducteur est tenu à distance à la fois du monde et des mots.

La traduction, qui trouve tant de résonnances dans la Loi 22 , semblerait pouvoir s'ordonner autour de principes admis par tous. De fait, pendant longtemps, on adhéra sans restriction à quelques articles de foi. Premièrement, on considérait la fidélité absolue au texte comme possible : le texte existait en dehors du traducteur et était facilement traité comme un objet; le langage du texte avait une signification littérale, qu'il était facile de restituer par des moyens lexicographiques. $\mathrm{Sa}$ signification devait être rendue par le traducteur en un langage faisant fi de l'esprit de la langue d'arrivée. Deuxièmement, en traitant un texte, le traducteur 
devait user dans son langage de la plus grande sobriété d'expression, qui proscrivait le « jargon », c'est-à-dire les termes techniques français, à moins bien sûr qu'ils ne soient suivis, entre parenthèses et en caractères gras, du terme anglais. Ces principes sont les évidences de la traduction traditionnelle, illustrés par des générations de secrétaires bilingues.

S'agit-il d'un simple conflit de générations? Au contraire des anciens, les nouveaux traducteurs sont presque tous de formation universitaire. En réalité, les conséquences de la Loi 22 pour la profession correspondent à une prise de conscience: une conception nouvelle de la traduction a été élaborée dans le cadre général du processus de francisation des entreprises. En effet, la conception traditionnelle de la traduction ne repose plus sur des évidences universelles. Déjà, dans nombre d'entreprises, l'appellation «service de traduction» a glissé vers celle de "service linguistique »; le phénomène se poursuivant, les services linguistiques se sont vus de plus en plus souvent confier la révision et même la rédaction de textes français. La traduction est dès lors réservée aux textes provenant de l'extérieur du Québec ou d'employés unilingues, suivant un ordre de priorité dicté par la diffusion du document au Québec.

On comprendra aisément ce qu'une telle évolution exige du traducteur. Pour réviser un texte ou participer à sa rédaction, il ne faut plus s'arrêter aux données immédiates, mais tenir compte de toutes les significations latentes, de «l'intentionalité inconsciente» de l'auteur. Le traducteur-rédacteur aura donc le souci de dégager les structures d'une ébauche ou d'une pensée : par un double mouvement d'analyse et de synthèse, il devra découvrir entre chaque partie du texte et sa totalité la relation d'interdépendance. Vue sous cet angle, la traduction n'a offert jusqu'ici qu'une seule méthode cohérente d'analyse : la recherche terminologique qui fiche les mots, les définitions et les contextes pour en assurer la normalisation puis la diffusion. Autrement, le traducteur reste confronté non à son propre langage, mais à un objet extérieur.

L'entreprise, pour sa part, entrevoit d'abord la francisation de ses activités québécoises sous deux aspects exclusifs : la traduction et les cours de langue seconde. L'ignorance naïve qu'elle a longtemps entretenue à l'endroit du fait français ne saurait l'inciter à agir autrement. D'où le paradoxe flagrant du rôle à confier aux traducteurs dans l'entreprise, dans le double but d'améliorer la qualité $\mathrm{du}$ français écrit et de réduire à ses justes proportions la «traductose » qui afflige le monde des affaires, et de la recrudescence sur le marché des traducteurs incompétents. Comme les Croisades, les vocations guerrières, la Loi 22 a suscité les vocations de traducteur. Il n'est de jour que n'apparaisse sur le marché un nouveau cabinet de traduction, formé de chevaliers d'industrie attirés par les meuglements de la vache à lait. Il est inadmissible que ces professionnels d'occasion viennent embrouiller davantage les cartes du français au Québec. Pour l'entreprise, ils présentent l'inconvénient de vendre parfois fort cher des services de mauvaise qualité, nuisant ainsi à son chiffre d'affaires et à sa bonne image; pour les vrais traducteurs, ils présentent celui tout aussi néfaste d'entretenir l'arbitraire injustifié dans lequel se débat encore leur profession. 
La Régie de la langue française a annoncé son intention de ne pas approfondir les problèmes de la traduction; c'est là un point de vue que nous comprenons tout à fait, compte tenu de l'énormité de la tâche qu'elle doit entreprendre. D'autre part, les traducteurs constituent, avec de rares rédacteurs, les seules ressources humaines dont dispose l'entreprise pour améliorer la qualité de ses communications écrites. Il va de l'intérêt de l'entreprise autant que de celui de la société québécoise et de son gouvernement que la profession de traducteur, eu égard aux intérêts des autres professionnels de la langue écrite, soit reconnue officiellement et, surtout, policée. Il serait souhaitable que la Société des traducteurs du Québec et la Régie de la langue française recherchent ensemble les moyens d'assurer la qualité du français au Québec dans l'esprit de cette Loi 22 qui ressemble encore à une terre peu parcourue et où manquent les chemins.

Michel Guillotte 